\title{
ADECUACIÓN Y ESTUDIO DE RESTOS ÓSEOS EN ANTROPOLOGÍA FORENSE
}

\author{
ADEQUACY AND STUDY OF SKELETAL REMAINS IN \\ FORENSIC ANTHROPOLOGY
}

\author{
Cristina M $\mathrm{M}^{\mathrm{a}}$ Beltrán Aroca, Eloy Girela López, \\ Luis Jiménez Reina, María Moreno Llorente, \\ Laura Linares González, Araceli Rodríguez Cano, \\ cristinabeltran@uco.es \\ Universidad de Córdoba
}

Received: 05/07/2017

Accepted: 04/12/2017

\begin{abstract}
Having demonstrated the importance of forensic anthropology in the positive identification of human skeletal remains, we consider it relevant to learn a basic management of the techniques by the students of the Medical Degree, in the context of the subject "Legal Medicine and Ethics". The main objective was to apply theoretical and technical knowledge on physical and forensic anthropology to identify real cases through group work dynamics, concluding with justified decision making. Sixteen 2-hour workshops were organized for groups of 8 students, using skeletal remains of 4 individuals mixed with animal bones. After the human remains were identified, the four skeletons were isolated and an inventory of the available bones was made. The basic biological profile of each of them was analyzed: sex, age and height. These data were entered in a protocol form, which they delivered at the end of the practice. Subsequently, they conducted an anonymous questionnaire of 10 items about the degree of satisfaction on contents, methodology, and utility in their training.

With a response rate of $78.4 \%(n=125)$, the highest mean scores were given to items related to: teacher and subject ( $\chi 4,98$; Dt. 0.14$)$, expository clarity $(\chi 4,94$, Dt. 0.24$)$ and adequacy of methodology $(\chi 4,92$, Dt. 0,28$)$. In addition to the favorable results obtained, it was objectified through the protocolized forms, the adequate resolution of real practical cases, based on a joint decision making and justified on the theoretical contents learned.
\end{abstract}

Keywords: Forensic anthropology; identification; group dynamics

\section{Resumen}

Demostrada la importancia de la antropología forense en la identificación positiva de restos óseos humanos, consideramos relevante el aprendizaje de un manejo básico de las técnicas por los estudiantes del Grado de Medicina, en el contexto de la asignatura "Medicina Legal y Ética". El objetivo principal era 
aplicar conocimientos teóricos y técnicas de antropología física y forense para identificación de casos reales mediante dinámicas de trabajo en grupo, concluyendo con una toma de decisiones justificada. Se organizaron 16 talleres de 2 horas de duración para grupos de 8 alumnos, utilizando restos esqueléticos de 4 individuos mezclados con huesos animales. Distribuidos por parejas, y una vez identificados los restos humanos, aislaron los 4 esqueletos realizando un inventario de los huesos disponibles, y una estimación básica del perfil biológico de cada uno de ellos: sexo, edad y estatura. Dichos datos fueron consignados en un formulario protocolizado de trabajo, que entregaron al finalizar la práctica. Posteriormente, realizaron un cuestionario anónimo de 10 preguntas, sobre el grado de satisfacción acerca de contenidos, metodología, y utilidad en su formación.

Con una tasa de respuesta del 78,4\% $(n=125)$ las puntuaciones medias más elevadas fueron otorgadas a los ítems relacionados con: profesor y materia ( $\chi$ 4,98; Dt. 0,14$)$, claridad expositiva ( $\chi 4,94$; Dt. 0,24) y adecuación de metodología $(\chi 4,92$; Dt. 0,28). Además de los resultados favorables obtenidos, se objetivó mediante los formularios protocolizados, la adecuada resolución de casos prácticos reales, basada en una toma de decisiones conjunta y justificada en base a los conocimientos teóricos aprendidos.

Palabras clave: Antropología forense; identificación; dinámica de grupo

\section{INTRODUCCIÓN}

La antropología forense es la rama de la antropología física que, por motivos forenses, se encarga de la identificación de restos que se sospecha que sean humanos y que están en avanzado estado de descomposición o en estado esquelético.

La identificación es un elemento esencial y primario de carácter jurídico que garantiza las relaciones correctas con las personas. Este proceso es una de las responsabilidades más importantes de la antropología y debe priorizarse en todos los casos, tanto por su trascendencia legal y judicial a efectos de la investigación en curso, como humanitaria atendiendo a las necesidades de las familias de las personas desaparecidas. Por ello, los huesos humanos se analizan con el fin de extraer la mayor cantidad posible de información acerca de las personas representadas por esos restos y las circunstancias en las que fallecieron.

En determinados casos la autoridad judicial decide que unos restos humanos requieren de investigación médico-legal, e inicialmente, uno de los primeros análisis de interés médico-legal y antropológico a realizar, es la identificación genérica y de la especie, es decir, establecer la naturaleza del material óseo, y diferenciar si son de origen animal o humano. En los casos en los que los restos no se encuentren especialmente deteriorados, es un estudio que ofrece pocas dificultades 
tras la limpieza del material, y se puede determinar con precisión la naturaleza del mismo.

A continuación, se encargará de analizar las características demográficas de la persona (sexo, edad, estatura, ancestro), una vez que el tejido blando del cuerpo humano se ha deteriorado a tal punto de que se hace imposible un reconocimiento visual.

Durante el análisis se intentará también determinar la presencia de evidencias traumáticas de distinta índole (herida de bala, contusiones, heridas cortantes...).

Además, se procurará determinar el tiempo transcurrido desde la muerte de la persona y el rescate o excavación de los restos humanos.

En definitiva, la antropología forense puede proveer información vital presente en el esqueleto, que ayude en la identificación positiva de la persona fallecida, así como otros datos de interés en relación a la causa de la muerte. Por estos motivos en los últimos años se ha puesto de manifiesto la importancia de dichas técnicas, por lo que consideramos relevante la aproximación al conocimiento de un manejo básico de las mismas por parte de los estudiantes.

\section{OBJETIVOS}

Objetivo principal: Aplicar conocimientos teóricos y técnicas de antropología física y forense para identificación de casos reales mediante dinámicas de trabajo en grupo, concluyendo con una toma de decisiones justificada

Objetivos específicos:

- Discriminar restos humanos de animales

- Cumplimentar formulario de trabajo, realizando un inventario de los huesos disponibles, y mediante el establecimiento del perfil biológico: fundamentalmente sexo, aproximación a la edad y estatura.

- Análisis de algunos casos forenses disponibles

\section{METODOLOGÍA}

\subsection{PREPARACIÓN Y ADECUACIÓN DE RESTOS ÓSEOS}

Procedimos a la recogida de los restos óseos pertenecientes a 4 individuos adultos y 2 infantiles, de sexo, edad y data de la muerte conocidos, procedentes de los cementerios de San Rafael y Ntra. Sra. De la Fuensanta (Córdoba) y posterior traslado en bolsas de plástico a las dependencias de la Facultad de Medicina y Enfermería (Figura 1A y B). Posteriormente realizamos la limpieza de los mismos, retirando las partes blandas adheridas mediante cepillos, detergente jabonoso y abundante agua (Figura 1C). Una vez eliminados los restos de tejidos 
blandos, los huesos fueron sumergidos en lejía diluida al 30\% durante 15 minutos, tras lo que fueron irrigados con abundante agua corriente con la finalidad de ser aclarados y evitar la precipitación y cristalización del hipoclorito de sodio en el interior del hueso (Figura 1D). Finalmente se procedió a su secado con aire caliente durante varios días sobre superficie plana.

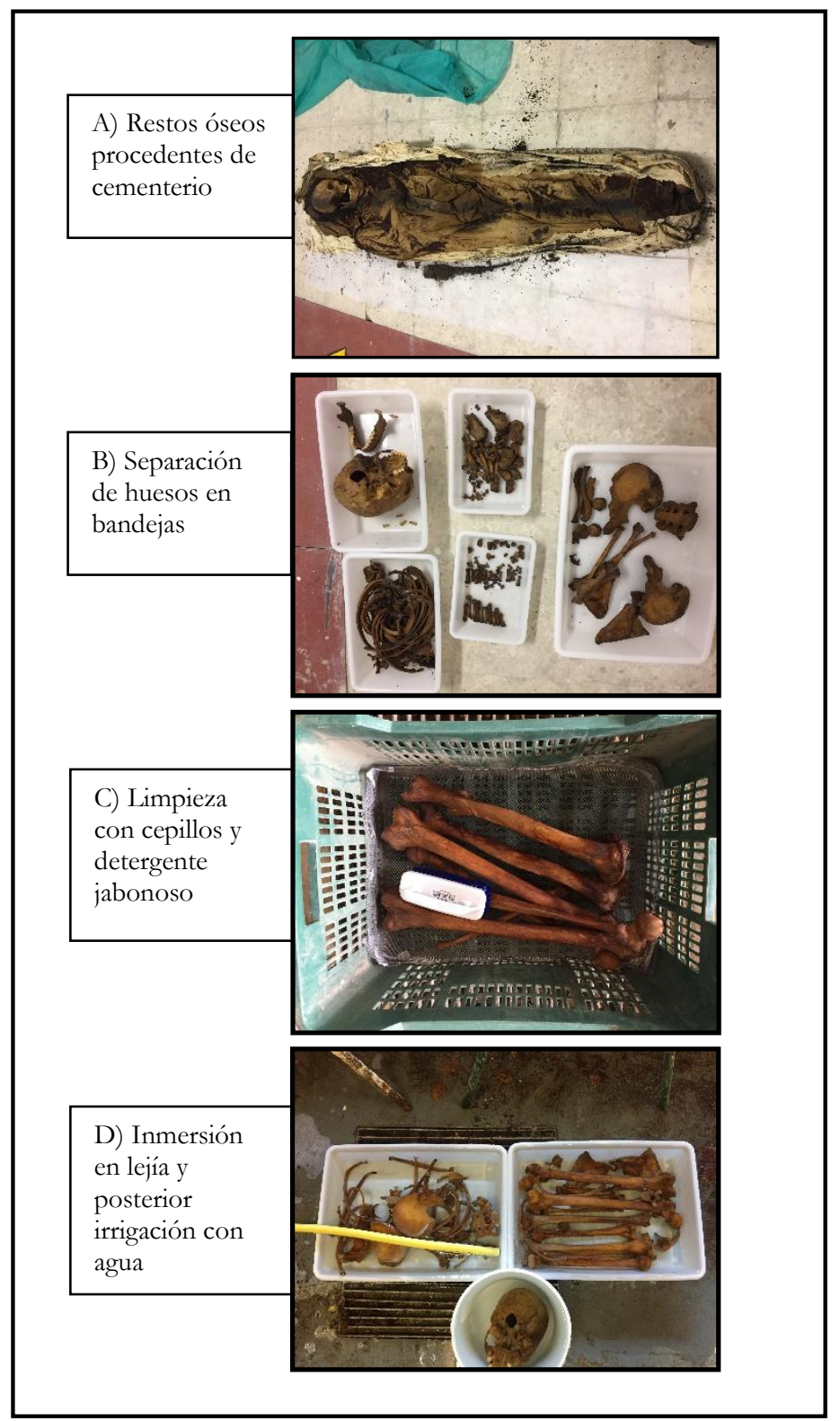


Figura 1. Proceso de limpieza y adecuación de restos óseos

\subsection{ORGANIZACIÓN DE TALLERES}

Una vez se encontraban los huesos limpios, organizamos 16 talleres de 2 horas de duración para grupos de 8 alumnos del grado de Medicina en la asignatura de Medicina Legal y Ética. Dichos restos se mezclaron con huesos animales. Distribuidos por parejas, y una vez identificados los restos humanos, aislaron los 4 esqueletos para realizar un inventario de los huesos disponibles, y una estimación básica del perfil biológico de cada uno de ellos: sexo, edad y estatura. Además de los restos óseos, utilizaron como material tablas ilustrativas con imágenes preparadas por nosotros, para la determinación de sexo y edad. Además en este último caso se ayudaron de unos modelos de sínfisis púbicas fabricados en resina France Casting ${ }^{\circledR}$ (Figura 2A) basados en el método de Suchey-Brooks (1990). Para el estudio de la estatura, la medición de los huesos largos se realizó mediante una tabla osteométrica (Figura 2B).

Los datos recogidos fueron consignados en un formulario protocolizado de trabajo, que entregaron al finalizar la práctica (Anexo I). De igual forma, debían analizar algunas cuestiones planteadas sobre casos forenses.

Finalmente, realizaron una encuesta anónima, previamente validada, de 10 preguntas $(1=$ malo, $2=$ regular, $3=$ aceptable, $4=$ bueno, $5=$ excelente), sobre el grado de satisfacción acerca de si los contenidos se ajustaban a sus expectativas, profundidad de los mismos, duración del taller, adecuación de la metodología empleada, lugar de realización del mismo, conocimientos y actitud del profesor y utilidad del taller. La última cuestión era de respuesta libre sobre aspectos a mejorar.

Los resultados fueron sometidos a un análisis descriptivo calculando frecuencias, medias aritméticas, rangos y desviaciones típicas, con el programa SPSS Statistics v. 19.

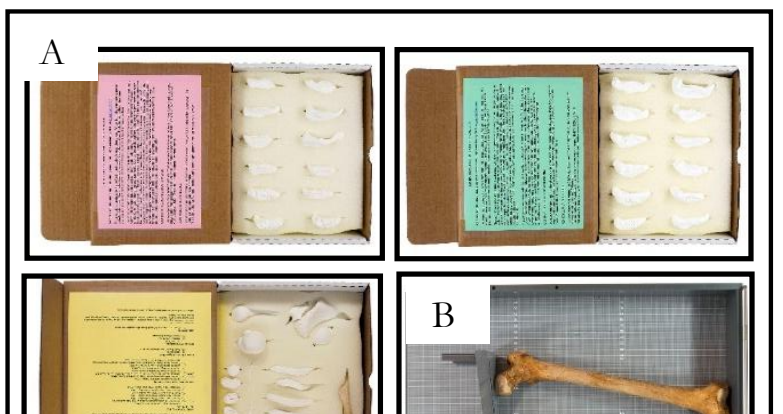


Figura 2. Material empleado para la determinación de la edad y estatura

\section{DESCRIPCIÓN}

Los talleres organizados se llevaron a cabo en la sala de Disección I de la Facultad de Medicina y Enfermería en grupos de hasta un máximo de 9 alumnos. La dinámica a seguir era fundamentalmente práctica aunque era necesario el apoyo previo a cada una de las actividades, con una presentación Power-Point, puesto que los conceptos teóricos a aplicar eran muy específicos dentro del área y no se habían explicado previamente. Debido a la limitación de tiempo (2 horas), se les concedía un tiempo limitado para la realización de cada una de las actividades, en torno a los 10-12 minutos en función de la complejidad.

En el centro de la sala se colocó una mesa central con diversos tipos de huesos humanos mezclados con restos animales, alrededor de la cual se disponía el grupo de estudiantes (Figura 3A).

\subsection{DIAGNÓSTICO DE ESPECIE Y EXTENSIÓN DE ESQUELETOS}

Tras una breve introducción sobre la importancia de la Antropología Física y Forense en el campo de la Medicina Legal, se colocaban por parejas en mesas situadas alrededor de la central, debiendo extender en cada una de ellas un esqueleto lo más completo posible con los huesos disponibles. Finalizada la actividad, se revisaban los esqueletos montados para corregir errores, siendo los más frecuentes los relacionados con lateralidad y posición (Figura 3B). A su vez, debían cumplimentar en el formulario de trabajo, un apartado específico a modo de Inventario óseo, marcando aquellos huesos presentes o ausentes, y el número de los mismos.

A continuación, reunidos de nuevo entorno a la mesa central, separábamos aquellos huesos diferentes al resto y pertenecientes a la especie animal, analizando las principales características diferenciales entre ambos en cuanto a la superficie cortical, morfología, así como el tamaño. En este último punto, reflexionábamos acerca de aquellos huesos animales de características similares a los humanos y las 
diferencias existentes con un individuo infantojuvenil de la especie humana. Idéntico procedimiento se realizó con dientes de distintas especies animales en función de la morfología, tamaño, superficie de masticación...

\subsection{ESTUDIO DEL PERFIL BIOLÓGICO}

Puesto que el tiempo para la realización de las siguientes actividades era limitado, las características del perfil biológico analizadas fueron las siguientes:

Estimación de sexo: se realizaba mediante análisis morfológico de huesos coxales y cráneo. Utilizando los huesos de la mesa central, y con el apoyo de imágenes, se les mostraban las características más dimórficas de ambos huesos para determinar el sexo del sujeto (Figura 3C). Posteriormente y en un tiempo determinado, debían analizar por parejas, los huesos de los esqueletos presentes en sus mesas de trabajo (Figura 3D).

Determinación de la edad: se introducían brevemente los diferentes métodos en función de la edad del sujeto (feto, infantil, juvenil, adulto). A continuación, ayudándose de los modelos de resina con edades preestablecidas del método Suchey-Brooks (1990) anteriormente descrito, comparaban la morfología y variaciones de la sínfisis púbica de su esqueleto, señalando el estadio más adecuado y por tanto el rango de edad aproximado (Figura 3E). Una vez más la falta de tiempo condicionó el no poder explicar suficientemente otros métodos que permitieran una mayor aproximación a la edad real del sujeto. Puesto que los casos a estudiar eran cuatro individuos adultos, posteriormente se les mostraban las diferencias con dos individuos infantiles de 2 meses y 8 años de edad respectivamente.

Estimación de estatura: como último aspecto importante del perfil, tras familiarizarse con los instrumentos de medida de huesos largos utilizados en antropología, realizaron mediciones del fémur y húmero derechos de cada uno de los esqueletos con la tabla osteométrica, aplicando las fórmulas de regresión del método De Mendonça (2000) para el cálculo de los valores máximo, mínimo y medio de la estatura (Figura 3F).

Cada una de estas características del perfil biológico -sexo, edad y estatura- fueron recogidas en los formularios de trabajo.

Como última tarea, estudiaron tres fragmentos de tres cráneos diferentes para análisis forense. Dos de ellos presentaban un orificio de entrada, correspondiente a un disparo perpendicular por arma de fuego 
(hueso occipital izquierdo y temporal derecho). El tercero (hueso parietal izquierdo) mostraba igualmente un orificio de entrada pero en un disparo oblicuo, permitiendo determinar además la dirección del mismo (Figura 3G). 


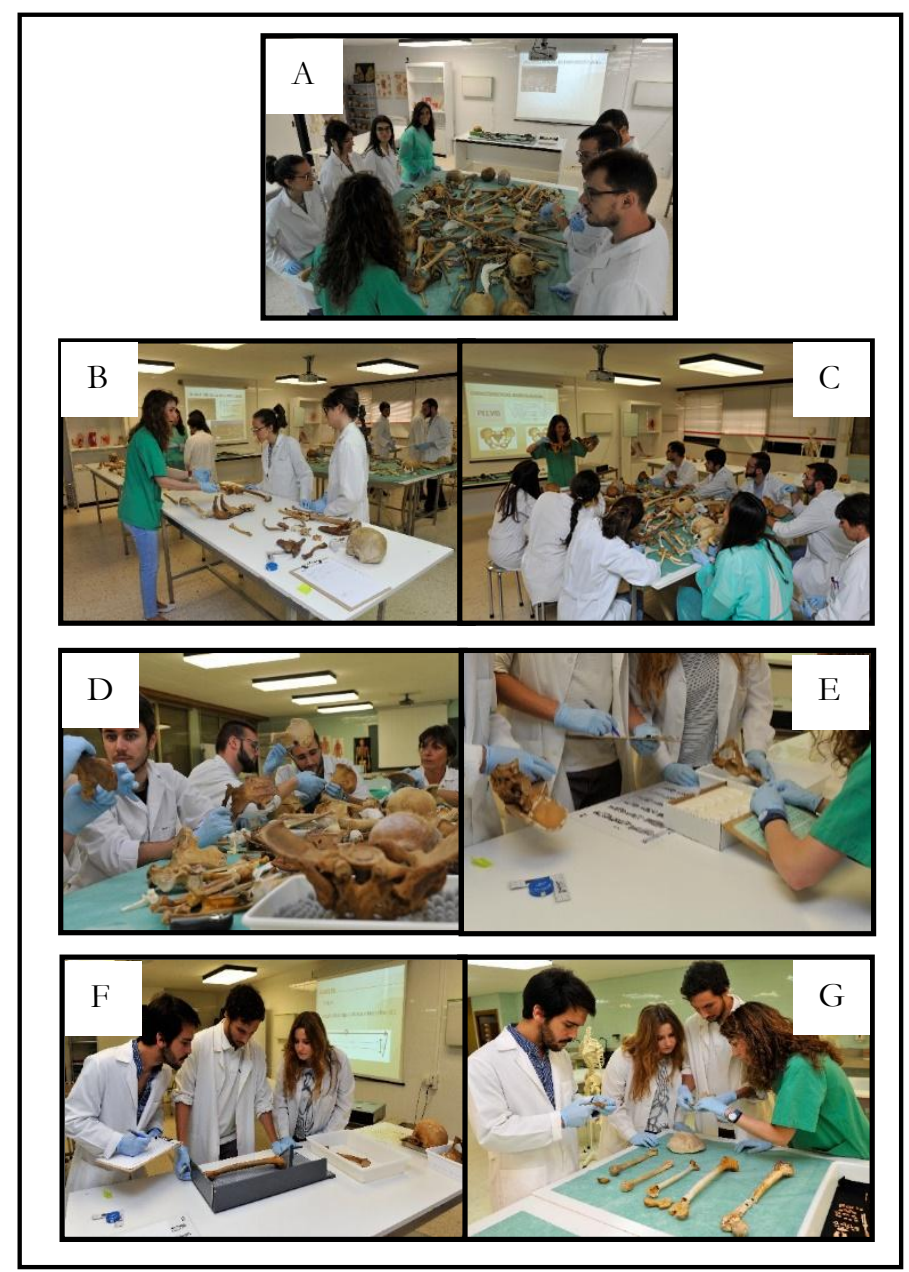

Figura 3. Desarrollo de cada una de las actividades

Finalmente realizaron un cuestionario anónimo de satisfacción (Anexo II) acerca del taller realizado a través de la plataforma moodle: (http://moodle.uco.es $/ \mathrm{m1617} / \mathrm{mod} /$ feedback/view.php?id=177456)

\section{RESULTADOS OBTENIDOS}

De un total de 125 alumnos asistentes al taller, la tasa de respuesta fue de un $78,4 \%(\mathrm{n}=98)$.

Las puntuaciones medias más elevadas (Figura 4) fueron otorgadas a los ítems relacionados con: profesor y materia $(\chi 4,98$; Dt. 0,14$)$, 
claridad expositiva $(\chi 4,94$; Dt. 0,24$)$ y adecuación de metodología $(\chi$ 4,92; Dt. 0,28).

Excepto en la utilidad del taller, donde la valoración más frecuente era buena, en el resto las calificaciones más frecuentes fueron de excelente (Figura 5).

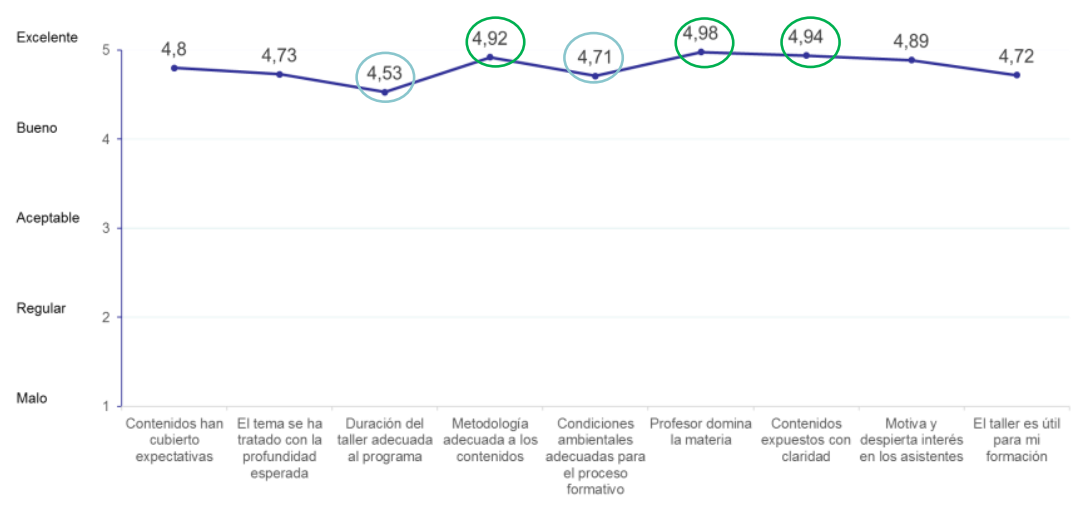

Figura 4. Puntuaciones medias otorgadas a cada uno de los ítems valorados en la encuesta

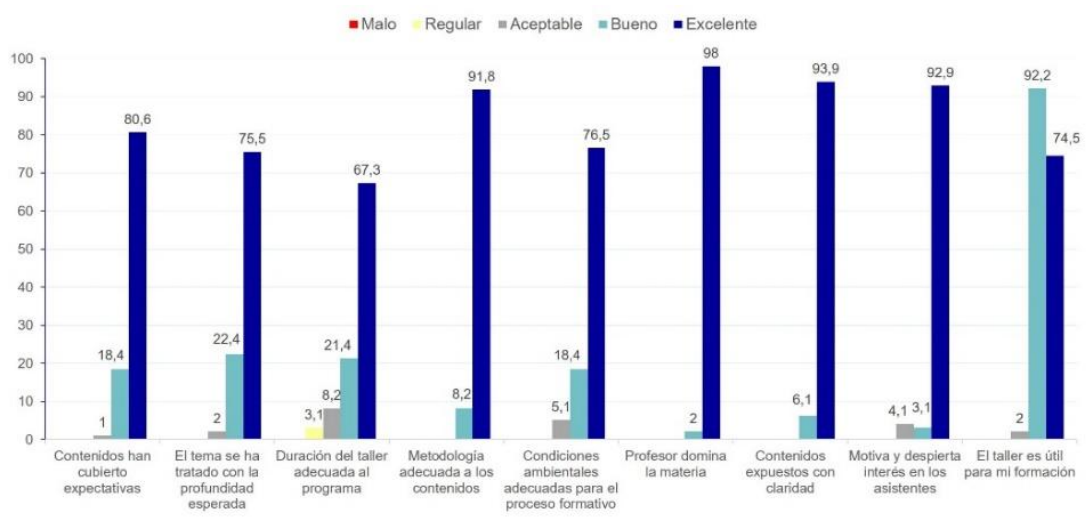

Figura 5. Frecuencias obtenidas en cada uno de los ítems valorados, según escala

Los resultados obtenidos fueron difundidos mediante una comunicación oral en el XIV Foro Internacional sobre la Evaluación de la Calidad de la Investigación y de la Educación Superior (FECIES) celebrado en Granada (22-24 de Junio, 2017) (Anexo III). 


\section{UTILIDAD}

El desarrollo del taller pretende el aprendizaje del trabajo en pequeños grupos sobre casos reales. La toma de contacto con las principales técnicas empleadas en antropología y su aplicación forense, permite su intervención en la identificación de restos óseos a estudio en casos básicos de antropología forense, así como la discriminación y potencial rendimiento de métodos y procedimientos para establecer posibles causas y circunstancias del fallecimiento.

A su vez, permitirá al alumno identificar el contexto jurídico y ético en el que se desarrollan las actuaciones en el ámbito de la antropología forense y determinar la trascendencia jurídico-penal de los hallazgos encontrados.

\section{CONCLUSIONES}

Los ítems valorados en los cuestionarios resultaron especialmente favorables en cuanto a la metodología y desarrollo del taller, si bien mejorables respecto a la duración del mismo y condiciones del aula.

De igual forma, mediante los formularios protocolizados fue posible objetivar una adecuada resolución de casos prácticos reales, basada en una toma de decisiones conjunta y justificada en base a los conocimientos teóricos aprendidos.

\section{AGRADECIMIENTOS}

The authors would especially like to thank Dr. Esteban Tarradas Merino for imaging technical support, Gonzalo Ruiz Roldán for helping in the adaptation and preparation of bone remains, Dr. Inmaculada Alemán Aguilera, from University of Granada, and the personnel of the Cemetery in Córdoba for providing us juvenile and adult skeletal remains.

\section{BIBLIOGRAFÍA}

BROOKS, S., SUCHEY J. "Skeletal age determination based on the os pubis: A comparison of the Acsádi-Nemeskéri and Suchey-Brooks methods", Human Evolution 1990/5, p. 227-238

BUIKSTRA, J.D., UBELAKER, D. (eds). Standards for data collection from buman skeletal remains, Arkansas, 1994.

CHRISTENSEN, A.M., PASSALACQUA, N.V., BARTELINK, E.J. (eds). Forensic Anthropology: Current Methods and Practice, USA, 2014. 
CUNNINGHAM, C., SCHEUER, L., BLACK, S. (eds). Developmental Juvenile Osteology, London, 2000.

DE MENDONÇA, M.C. "Estimation of height from the length of long bones in a Portuguese adult population", American Journal of Physical Anthropology 2000/112, p. 39-48.

ISCAN, M.Y., STEYN, M. The Human Skeleton in Forensic Medicine, Springfield, 2013.

UBELAKER, D. Human Skeletal Remains. Excavation, analysis, interpretation, Washington, 1989.

VILLALAÍN, J.D. Identificación antropológica policial y forense, Madrid, 2000. WHITE, T.D., FOLKENS, P.A. The Human Bone Manual, London, 2005. 